\title{
Comparison of Unsatisfactory Rates and Detection of Abnormal Cervical Cytology Between Conventional Papanicolaou Smear and Liquid-Based Cytology (Sure Path ${ }^{\circledR}$ )
}

\author{
Saroot Kituncharoen $^{1}$, Patou Tantbirojn ${ }^{2 *}$, Somchai Niruthisard ${ }^{2}$
}

\begin{abstract}
Purpose: To compare unsatisfactory rates and detection of abnormal cervical cytology between conventional cytology or Papanicolaou smear (CC) and liquid-based cytology (LBC). Materials and Methods: A total of 23,030 cases of cervical cytology performed at King Chulalongkorn Memorial Hospital during 2012-2013 were reviewed. The percentage unsatisfactory and detection rates of abnormal cytology were compared between $\mathrm{CC}$ and LBC methods. Results: There was no difference in unsatisfactory rates between CC and LBC methods $(0.1 \%$ vs. $0.1 \%, p=0.84)$. The detection rate for squamous cell abnormalities was significantly higher with the LBC method $(7.7 \%$ vs. $11.5 \%, p<0.001)$, but those for overall abnormal glandular epithelium were similar $(0.4 \%$ vs. $0.6 \%, p=0.13)$. Low grade squamous lesion (ASC-US and LSIL) were more frequently detected by the LBC method $(6.1 \%$ vs. $9.5 \%, p<0.001)$. However, there was no difference in high gradd squamous lesions (1.1\% vs. 1.1\%, $p=0.95)$. When comparing between types of glandular abnormality, there was no significant difference the groups. Conclusions: There was no difference in unsatisfactory rates between the conventional smear and LBC. However, LBC could detect low grade squamous cell abnormalities more than CC, while there were similar rates of detection of high grade squamous cell lesions and glandular cell abnormalities.
\end{abstract}

Keywords: Cervical cancer screening - conventional cytology - detection rate - liquid based cytology - unsatisfactory rate

Asian Pac J Cancer Prev, 16 (18), 8491-8494

\section{Introduction}

Cervical cancer is the second most common cancer and cause of death among women worldwide, especially in developing country (Canda, et al., 2009). In Thailand, the leading cancer in female population is also cervical cancer (Wilailak, 2009). Morbidity and mortality of cervical cancer can be reduced by early detection of precancerous lesion. In present, there are several methods of cervical cytology screening, such as cervical cytology and human papilloma virus (HPV) test. Among these tests, cervical cytology is widely used because it is easily performed and causes less expense. The cervical cytology can be divided into two types: conventional cytology (CC) and liquid based cytology (LBC).

The conventional cytology, or commonly known as papanicolaou or Pap test, has been used in screening for cervical cancer since the 1940s (Kirschner, et al., 2006). It is easily done by direct transfer collected cells from the cervical transformation zone to microscopic slide which will subsequently fixed in alcohol and sent to laboratory for staining and evaluation. However, the accuracy of this screening tool widely varies. The sensitivity is $30 \%$ to $87 \%$, while the specificity is $86 \%$ to $100 \%$ (Sylvia, et al., 2006).

Liquid-based cytology has been introduced in mid1990s. LBC is slightly more complicated in processing and more expensive than CC. After the cervical cells are collected with a sampling device and rinsed into vial with preservation solution, the whole vial will be sent to laboratory for process the slides preparation by automated equipment. It has been reported that the LBC method can significantly reduce in the number of unsatisfactory specimens from $4.3-11.5 \%$ by CC to only $0.3-1.7 \%$ by LBC (Davey, et al., 2006, Akamatsu, et al., 2012, Singh, et al., 2015). Several studies have shown that LBC has possibly higher rate of high grade squamous intraepithelial lesion (HSIL) and glandular abnormalities detection (Kirschner et al., 2006, Schledermann, et al., 2006, Halford, et al., 2010, Burnley, et al., 2011). However, some studies have shown equal or decreased sensitivity and specificity of LBC, comparing to CC.

Based on these benefits, many developed countries have switched from CC to LBC for cervical cancer screening program. Nowadays, LBC has much decreasing in expense and can be implemented in a routine screening service. However, as the benefits of LBC are still inconclusive, the present study was conducted to compare 


\section{Saroot Kituncharoen et al}

unsatisfactory rates and detection rates of abnormal cervical cytology between these two methods.

\section{Materials and Methods}

This was a retrospective study. After approval from the Institutional Review Board of the Faculty of Medicine, Chulalongkorn University; the cytologic report and clinical data of women that underwent screening cervical cytology at King Chulalongkorn Memorial Hospital during 2012-2013 were reviewed. For CC, sample of cervical cells was taken by Ayre's spatula and smeared on standard glass slide. LBC samples were collected by broom and preserved in Surepath ${ }^{\circledR}$ solutions. Both CC and LBC specimens were prepared by cytotechnologists. All cases reposted as unsatisfactory specimen and those with cytologic abnormality were reviewed by experienced pathologists to confirm the diagnosis. The satisfactory cases with negative for intraepithelial lesion or malignancy were randomly reviewed. The criteria outlined by Bethesda system 2014 was used to interpret the satisfactory of specimen and classification of cytology. The results of cervical cytology were correlated with menstruation status of the women. The cases with high graded lesion and carcinoma were also correlated with follow-up specimen from biopsy or excisional procedures as possible.

All statistical analysis was performed using the Statistical Package for Social Sciences (SPSS) versions 17 for Windows (SPSS, Chicago, IL, USA). Pearson Chisquare test and exact test were used to analyze the data when appropriate. $P$ value of 0.05 or less was considered to be statistical significance.

\section{Results}

Of total 23,030 specimens, 11,179 specimens were CC, while 11,851 specimens were LBC. Women in both groups had similar mean age (CC vs. LBC, 46.7 vs. 46.9 years) and postmenopausal status (CC $41.5 \%$,

Table 1. Comparison of Detection Rate Between CC and LBC

\begin{tabular}{lrrr}
\hline Results & \multicolumn{1}{c}{ CC } & \multicolumn{1}{l}{ LBC } & p-value \\
& $(\mathrm{n}=11,179)$ & $(\mathrm{n}=11,851)$ & $<0.001$ \\
\hline NILM & $10,260(91.8 \%)$ & $10,397(87.7 \%)$ & $<0.001$ \\
Abnormal cytology & $906(8.1 \%)$ & $1,442(12.3 \%)$ & $<0.001$ \\
·Squamous cell lesions & $857(7.7 \%)$ & $1,366(11.5 \%)$ & 0.13 \\
-Glandular lesions & $49(0.4 \%)$ & $76(0.6 \%)$ & \\
\hline
\end{tabular}

Table 2. Comparison of Each Diagnosis between CC and LBC

\begin{tabular}{lccc}
\hline Results & $\begin{array}{c}\text { CC } \\
(\mathrm{n}=11,179)\end{array}$ & $\begin{array}{c}\text { LBC } \\
(\mathrm{n}=11,851)\end{array}$ & $\mathrm{p}$-value \\
\hline Squamous cell lesions & & & $<0.001$ \\
·ASC-US & $491(4.4 \%)$ & $755(6.4 \%)$ & $<0.001$ \\
·ASC-H & $53(0.5 \%)$ & $115(1.0 \%)$ & $<0.001$ \\
· LSIL & $189(1.7 \%)$ & $366(3.1 \%)$ & 0.13 \\
·HSIL & $84(0.8 \%)$ & $112(0.9 \%)$ & 0.003 \\
· Squamous cell carcinoma & $40(0.4 \%)$ & $18(0.2 \%)$ & 0.16 \\
Glandular lesions & $45(0.4 \%)$ & $64(0.5 \%)$ & 1 \\
·AGC & 0 & $1(0.01 \%)$ & 0.15 \\
·AIS & $4(0.03 \%)$ & $11(0.1 \%)$ & \\
·Adenocarcinoma & &
\end{tabular}

Table 3. Results of Follow-up Specimen from Biopsy or Excisional Procedures in High Grade Squamous (HSIL and Squamous Cell Carcinoma) and Glandular (AIS and Adenocarcinoma) Lesions

\begin{tabular}{llcr}
\hline Cervical cytology results & Histologic results & CC & LBC \\
\hline HSIL & & $(\mathrm{n}=72)^{*}$ & $(\mathrm{n}=102)^{*}$ \\
& Benign & $14(19.4 \%)$ & $31(30.4 \%)$ \\
& CIN1 & $9(12.6 \%)$ & $9(8.8 \%)$ \\
& CIN2-3 & $35(48.6 \%)$ & $37(36.3 \%)$ \\
Squamous cell carcinoma & Carcinoma & $14(19.4 \%)$ & $25(24.5 \%)$ \\
& & $(\mathrm{n}=34)^{*}$ & $(\mathrm{n}=16)^{*}$ \\
& Benign & $3(8.8 \%)$ & $2(12.5 \%)$ \\
AIS & CIN2-3 & $7(20.6 \%)$ & $3(18.7 \%)$ \\
& Carcinoma & $24(70.6 \%)$ & $(\mathrm{n}=1)$ \\
Adenocarcinoma & & $(\mathrm{n}=0)$ & $1(100 \%)$ \\
& Carcinoma & 0 & $(\mathrm{n}=111)$ \\
& & $(\mathrm{n}=4)$ & 0 \\
\end{tabular}

*Only cases with available specimen from biopsy or excisional procedures 
LBC $43.6 \%$ ). The unsatisfactory rate of $\mathrm{CC}$ was not significantly higher in $\mathrm{CC}(0.12 \%$ vs. $0.10 \%, \mathrm{p}=0.84)$. When correlate to menopausal status, the unsatisfactory rate in postmenopausal women was increased in $\mathrm{CC}$, but without statistically significance $(92.3 \% \mathrm{vs} .75 .0 \%$, $\mathrm{p}=0.52)$.

The overall detection rate of abnormal cytology was significantly higher in LBC (12.3vs. 8.1, p<0.001). Squamous cell abnormalities were more significantly detected in LBC $(7.7 \%$ vs. $11.5 \%, \mathrm{p}<0.001)$, but the detection rates of overall abnormal glandular epithelium were similar between both methods $(0.4 \% \mathrm{vs} .0 .6 \%$, $\mathrm{p}=0.13$ ) (Table 1). The low graded squamous lesion (ASCUS and LSIL) were more detected by LBC methods ( $6.1 \%$ vs. $9.5 \%, \mathrm{p}<0.001$ ) (Table 2). HSIL was significantly detected in LBC, while squamous cell carcinoma was significantly detected in CC. However, there was no difference when combined HSIL and squamous cell carcinoma into high graded squamous lesion $(1.1 \%$ vs. $1.1 \%, \mathrm{p}=0.95)$. When comparing between types of glandular abnormality, there was no significant difference among each group.

In high graded squamous lesions (HSIL and squamous cell carcinoma), the results of follow-up specimen from biopsy or excisional procedures were shown in Table 3. In HSIL, the final histologic diagnosis of carcinoma was higher in LBC, but with no statistically significance ( $24.5 \%$ vs. $19.4 \%, \mathrm{p}=0.21$ ). The final histologic diagnosis of carcinoma was similar in both groups if the result of the previous cervical cytology was squamous cell carcinoma.

\section{Discussion}

$\mathrm{CC}$ is a very good method for cervical cancer screening as it needs non-expensive equipment for processing and evaluation, which is suitable for the developing countries that have a very limited resource. Several studies reported the advantage of LBC over CC and suggested to convert from CC to LBC. However, there also are various studies indicated the similar sensitivity and specificity of these two methods. Besides of advantages of LBC in reduced the obscuration and smaller area to be evaluated, the most striking benefit of LBC is the ability to performed further tests such as HPV testing from the residual specimen. Currently, LBC is much cheaper than in the past, so it is possible to be used as routine service in developing country like Thailand. We gradually converted from CC to LBC for routine service during 2012-2013. This study was conducted to focus whether LBC can really improve the satisfactory and detection rates in our setting.

Several studies showed that LBC can significantly improve the unsatisfactory rate (Davey, et al., 2006, Akamatsu, et al., 2012, Singh, et al., 2015). In contrast, the unsatisfactory rate was similar between $\mathrm{CC}$ and LBC in our study. Two randomized clinical trials in the Netherlands and Italy, comparing the rate of unsatisfactory cervical cell samples in LBC and CC, reported there was a significant lower percentage of unsatisfactory in LBC $(0.33 \%$ vs. $1.11 \%$ and $2.59 \%$ vs. $4.10 \%$ ) (Castle, et al., 2010). Both studies showed a decrease in the unsatisfactory results by age group in LBC. In our study, when correlate to menopausal status, the unsatisfactory rate in postmenopausal women was also lower in LBC, but without statistically significance.

In the present study, the overall detection rate of abnormal cytology was significantly higher in LBC, especially with squamous cell abnormalities. The low graded squamous lesion (ASC-US and LSIL) were more detected by LBC methods. This finding is consistent with other studies. Fremont-Smith et al reported that the SurePath method was found to provide a statistically significantly greater detection rate for low-grade squamous intraepithelial lesion, including ASC-US, compared with conventional slides (Fremont-Smith, et al., 2004). Schledermann et al. (2006) also showed higher detection rate of mild dysplasia in LBC (Schledermann, et al., 2006). These results may be due to the more clarity of cells with reduced obscuring factors in LBC method.

Interestingly, we found that HSIL was significantly detected in LBC, while squamous cell carcinoma was significantly detected in CC. However, there was no difference when combined HSIL and squamous cell carcinoma into high graded squamous lesion. In a study by Akamatsu et al. (2012) of 236,511 cases in Japan, squamous cell carcinoma and HSIL detection rates were higher in LBC than CC (0.57\% vs. $0.25 \%)$ (Akamatsu, et al., 2012). In contrast, a study by Halford et al. (2010) demonstrated that the diagnosis of high grade lesion was not statistically significant between CC and LBC (Halford, et al., 2010). The finding in our study may be explained by the loss of tumor diathesis background in LBC which is very helpful in diagnosis of carcinoma. Therefore, the final histologic diagnosis of carcinoma was similar in both CC and LBC if the result of the previous cervical cytology was squamous cell carcinoma.

Burnley et al reported that $\mathrm{LBC}$ can improve detection of endocervical lesions with no significant increase in the number of reported cytological abnormalities suggesting endometrial origin (Burnley, et al., 2011). In the present study, the detection rates of overall abnormal glandular epithelium were similar between both methods. However, our study was limited by the very small numbers of cases with abnormal glandular epithelial cells. More prospective study was needed.

In conclusion, there was no difference in unsatisfactory rates between the conventional smear and LBC. LBC could detect low graded squamous cell abnormalities more than $\mathrm{CC}$, while there was similar rate of detection in high graded squamous cell lesion and glandular cell abnormalities. This study confirmed that CC is still a good method for cervical cancer screening.

\section{References}

Akamatsu S, Kodama S, Himeji Y, et al (2012). A comparison of liquid-based cytology with conventional cytology in cervical cancer screening. Acta Cytol, 56, 370-4

Burnley C, Dudding N, Parker M, et al (2011). Glandular neoplasia and borderline endocervical reporting rates before and after conversion to the Sure Path(TM) liquid-based cytology (LBC) system. Diagn Cytopathol, 39, 869-74

Canda MT, Namik D, Orcun S, et al (2009). Clinical results 


\section{Saroot Kituncharoen et al}

of the liquid-based cervical cytology tool, liqui-preptm, in comparison with conventional smears for detection of squamous cell abnormalities. Asian Pac J Cancer Prev, 10, 399-402

Castle PE, Bulten J, Confortini M, et al (2010). Age-specific patterns of unsatisfactory results for conventional Pap smears and liquid-based cytology: data from two randomised clinical trials. Br J Obstets Gynecol, 117, 1067-73

Davey E, Barratt A, Irwig L, et al (2006). Effect of study design and quality on unsatisfactory rates, cytology classifications, and accuracy in liquid-based versus conventional cervical cytology: a systematic review. Lancet, 367, 122-32

Fremont-Smith M, Marino J, Griffin B, et al (2004). Comparison of the SurePath liquid-based Papanicolaou smear with the conventional Papanicolaou smear in a multisite direct-to-vial study. Cancer, 102, 269-79

Halford JA, Batty T, Boost T, et al (2010). Comparison of the sensitivity of conventional cytology and the ThinPrep Imaging System for 1,083 biopsy confirmed high-grade squamous lesions. Diagn Cytopathol, 38, 318-26

Kirschner B, Simonsen K, Junge J (2006). Comparisons of conventional papanicolaou smear and surepath liquid-based cytology in the copenhagen population screening programme for cervical cancer. Cytopathol, 17, 187-94

Schledermann D, Ejersbo D, Hoelund B (2006). Improvement of diagnostic accuracy and screening conditions with liquidbased cytology. Diagn Cytopathol, 34, 780-5

Singh VB, Gupta N, Nijhawan R, et al (2015). Liquid-based cytology versus conventional cytology for evaluation of cervical Pap smears: experience from the first 1000 split samples. Indian J Pathol Microbiol, 58, 17-21

Sylvia T, louise K, William D, et al (2006). Direct comparison of liquid-based and conventional cytology in a South African screening trial. Int J Cancer, 118, 957-62

Wilailak S (2009). Epidemiologic report of gynecologic cancer in Thailand. J Gynecol Oncol, 20, 81-3 